\title{
Channel Allocation and Performance Study for the Integrated GSM/GPRS System
}

\author{
Huei-Wen Ferng and Yi-Chou Tsai \\ Department of Computer Science and Information Engineering, \\ National Taiwan University of Science and Technology, \\ Taipei 106, Taiwan \\ E-mail: hwferng@mail.ntust.edu.tw; D8915001@mail.ntust.edu.tw
}

\begin{abstract}
We propose dynamic channel allocation schemes which employ techniques such as channel reservation, service priority and queueing strategies (with or without threshold control) etc. Using these techniques allows system designers to differentiate Quality of Service (QoS) requirements (e.g., new call blocking/handoff forced termination probability and delay) required by different types of service. Via the simulation approach, the improvements offered by different strategies under different parameter sets and different network situations are investigated. The results obtained in this paper provide system designers guidelines of how to provide an excellent channel allocation scheme and how to choose suitable parameters in the integrated GSM and GPRS system.
\end{abstract}

\section{INTRODUCTION}

As time goes and technologies evolve, multimedia transmission has launched one of the main research topics in the contemporary and future research areas. Therefore, transmission technology has the trend to integrate voice, video, and data simultaneously in one system. In wired networks, Asynchronous Transfer Mode (ATM) [10] is one of the technologies aiming at this goal. As for wireless networks, we also have witnessed the current mobile communication systems, e.g., Generalized Packet Radio Service (GPRS) [5] and the future mobile communication systems, e.g., the third generation (3G) system [6] (or even in the beyond 3G (B3G) system [14] or in the fourth generation (4G) system [12]) offer integrated services to satisfy diverse requirements of different users who want to run multimedia applications on their own handsets.

Due to limited channels/resources in wireless networks, one of the most important issues in the field of resource management is how to efficiently make use of available channels/resources to achieve the best system performance. In the literature, most of past research has focused on this issue in cellular networks and Personal Communications System (PCS) or Personal Communications Network (PCN). For example, [19] addressed the dynamic priority queueing in PCS with voice service only. In [18], the authors addressed the channel allocation schemes for the voice and data integrated cellular systems to support multimedia applications. In voice and data integrated cellular such as Global System for Mobile communications (GSM) [4]/ PCS networks, the switching technology employed is circuit-switching which offers an inefficient way to carry data traffic which possesses more bursty nature; hence, the packet-switched GPRS is proposed to gain high efficiency in transmitting bursty data traffic. The packet-switching makes the resource allocation issue in GSM/GPRS different from that in cellular and PCS since the channel allocation schemes previously proposed for cellular and PCS do not distinguish different requirements in bandwidth between voice and data services, i.e., the slot numbers allocated for voice and data requests are equal; however, the number of slots allocated for data in GPRS is flexible based on the demand-basis. In the past, only a few papers have addressed this issue in the integrated GSM/GPRS system, e.g., [8], [9], [11], [13], [15], [17]. [8] tried to identify that how many channels should be allocated to GPRS sessions using the Markov-Modulated Poisson Process (MMPP) to model data traffic. [9] compared the performance of several proposed channel allocation schemes and the authors suggested that a dynamic channel allocation scheme with queueing (for both new voice and handoff requests) has better performance. [11] studied the issue for GSM-GPRS over a Low-EarthOrbit (LEO) satellite system. In [13] and [15], the authors employed an analytical approach and a simulation tool called GPRSsim, respectively, to study the performance of channel allocation schemes. [17] proposed two algorithms for channel allocation schemes, i.e., First Fit (FF) and Best Fit (BF), and used the simulation approach to study the performance under simplified assumptions.

In this paper, we also study the channel allocation schemes for the integrated GSM/GPRS system using the simulation approach to investigate in detail the improvements of techniques such as channel reservation, service priority, queueing strategies etc. The results obtained can serve guidelines to system designers on how to provide an excellent channel allocation scheme and how to choose suitable parameters in the integrated GSM and GPRS system. Among the related papers mentioned above, [9] is the closest work to ours. However, our paper differs from [9] in the following aspects: 1) Buffers are used for both voice and data requests while [9] provides a buffer for voice requests only. 2) Channel reservation, service priority, and threshold control of the buffer space are taken into consideration to further differentiate the QoS perceived by original and handoff requests, while these techniques are not considered in [9]. 


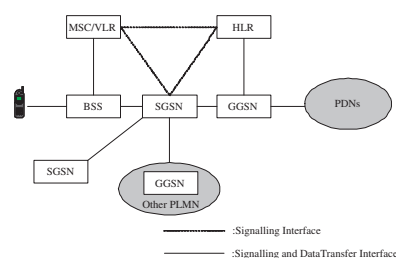

Fig. 1. The GPRS architecture.

The rest of the paper is organized as follows. In Section II, we briefly review the GPRS and introduce different channel allocation schemes. In Section III, the system model and assumptions used in simulation experiments are discussed. Section IV gives numerical examples and discussions on performance evaluation for different channel allocation schemes under different parameters and different network situations. Finally, Section V concludes the paper and outlines possible future directions.

\section{GPRS AND Channel Allocation Schemes}

For readers' convenience, we first extract and briefly review some concepts of GPRS. Readers may be referred to [5] for details about GPRS and [4] for GSM which is not described in this paper. Although data services have been provided in conventional circuit-switched GSM such as Short Message Service (SMS) [4] and High Speed Circuit-Switched Data (HSCSD) [4]; however, these services incur long access time due to call connection setup which may need more than a few seconds and offer a lower data date at $9.6 \mathrm{Kbps}$. These make SMS and HSCSD inefficient to carry bursty data traffic such as traffic in Internet. In contrast to SMS and HSCSD, GPRS affords users higher data rates (about 40-50 Kbps) and lower access time (lower than one second) using packetswitched technology. To integrate with existing GSM, GPRS modifies a few network elements in GSM and introduces two new GPRS Support Nodes (GSNs), i.e., Serving GPRS Support Node (SGSN) and Gateway GPRS Support Node (GGSN), where an SGSN is responsible for communications to and from Mobile Stations (MSs) while a GGSN acts as an interface to external Packet Data Networks (PDNs) such as Internet and X.25. Shown in Fig. 1 is the GPRS architecture. In the integrated GSM/GPRS system, the radio resources/channels in a cell are shared by both GSM and GPRS mobile users, but the allocation mechanism for GSM differs from that for GPRS. For GSM, a physical channel is

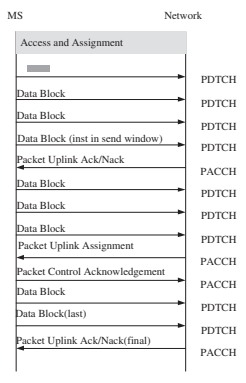

Fig. 2. Allocation of uplink data transfer.

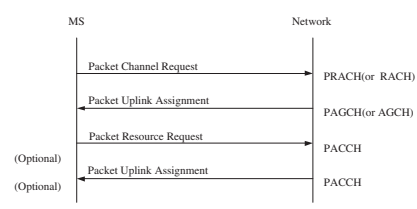

Fig. 3. An example of dynamic allocation of uplink data transfer.

permanently allocated to a MS during the entire call period, while multiple physical channels which are logically called Packet Data Traffic Channels (PDTCHs) may be allocated to a MS based on a demand basis for GPRS (which is called capacity on demand [5], i.e., channels are allocated when needed and released when completed). Moreover, multiple MSs of GPRS may share the same physical channel at a time. In GPRS, both Fixed Channel Allocation (FCA) and Dynamic Channel Allocation (DCA) schemes are allowed and the allocation for uplink and downlink is separate. Figs. 2 and 3 illustrate the uplink allocation and an example of dynamic allocation of uplink data transfer, respectively. An analogous procedure for downlink can be refereed to [5].

In the literature, either in circuit-switched cellular system or PCS or in the hybrid circuit- and packet-switched GSM/GPRS, the techniques frequently employed by resource allocation schemes to get better system performance include: 1) channel reservation, 2) service priority, and 3) queueing strategies with/without threshold control. The channel reservation scheme have been taken into consideration in [2], [3], [7], [18] etc. In particular, if a fixed number of channels is reserved for specific services, then such type of reservation is also known as guard channel, e.g., [7] or cutoff priority, e.g., [3]. If the number reserved can alter according to changes of network situation, one prefers using reservation/ dynamic reservation, e.g., [2] to reflect the dynamic nature. In [1], [2], [18], [19] service priority technique is used to improve system performance when dealing with resource allocation issue. As for the technique of queueing, [1], [2], [9], [18] have incorporated it into resource allocation to reduce blocking/forced termination probability. If multiple classes of requests are queued in the same buffer, different level of thresholds can be set to further adjust/discriminate QoS requirements, for example, [1] used such threshold control to get different blocking (with respect to new voice call) and forced termination (with respect handoff voice call) probabilities. Briefly speaking, channel reservation and service priority offer, respectively, different levels of available channels and different precedences to different service requests without throttling the request input rates while queueing strategy provides an extra buffer space for temporal request queueing. If threshold control is further utilized, the input rates allowed for different requests will be different; therefore, QoS perceived is also discriminated.

In this paper, the difference between FCA and DCA is emphasized on the number of slots to be allocated to data users. If this number is fixed, then the scheme is called FCA, while DCA means that the number of slots is flexible/adjustable. Since [9] has pointed out that DCA outperforms FCA and DCA with queueing strategies has the best performance; 
therefore, we will focus on DCA with finite queues at least (There are two buffers: one with size of $B_{v}$ for new voice call and handoff voice call and the other with size of $B_{d}$ for data packets) and other techniques to be further employed in this paper. Now we classify DCA's according to different techniques into the following categories:

- $D C A_{N Q P_{N C}}$ is a dynamic channel allocation scheme which is able to receive an amount of time slots with maximum value $K$ (a prescribed parameter), where ${ }_{N Q P}$ indicates no queueing priority among voice and data requests and ${ }_{N C}$ represents no buffer threshold control for the voice buffer (in which new voice and handoff voice requests are queued).

- $D C A_{Q P_{N C}}$ is similar to $D C A_{N Q P_{N C}}$ except voice calls (new call and handoff call) possess priorities higher than data packets.

- $D C A_{Q P_{T C}}$ is similar to $D C A_{Q P_{N C}}$ but buffer threshold control is employed for original voice request (with the maximum allowable number of original voice calls $T_{v}$ ).

- $D C A_{Q H P_{N C}}$ is similar to $D C A_{Q P_{N C}}$ but service priority given to handoff calls is higher than service priority given to the original calls.

- $D C A_{Q P G_{N C}}$ is similar to $D C A_{Q P_{N C}}$ but a portion of channels $C_{G}$ is reserved for handoff voice call among channels in a cell.

\section{SySTEM MODEL AND ASSUMPTIONS}

For simplicity, we assume the total number of channels in the channel pool shared by both GSM and GPRS users is fixed and same for each cell and denoted by $C$, i.e., the cell channel assignment under consideration is the fixed channel assignment [16]. Furthermore, each user moves to the adjacent cells in a uniform manner, i.e., the probabilities of user moving to any adjacent cell are equal. All above assumptions make cells homogeneous.

To capture the dynamic nature, we adopt the stochastic approach to model traffic patterns of the system and cell dwelling models etc. We assume that GSM (new) voice call and GPRS (new) data packet arrive according to Poisson distributions with rates $\lambda_{v}$ and $\lambda_{d}$, respectively. And the GSM (new and handoff) voice call holding time, GPRS packet transmission time, GSM user dwelling time follow the exponential distributions with mean $1 / \mu_{v}, 1 / \mu_{d}, 1 / \eta_{v}$, respectively. In this paper, we assume that data users are all static for simplicity (though this assumption may not sound reasonable in the real world).

\section{Performance Study AND Numerical ExAmples}

This section employs a simulation approach to study the improvements for different channel allocation schemes in terms of the following measures: new voice call blocking probability $P_{v, b}$, forced termination probability of handoff voice call $P_{h, f}$, data packet dropping probability $P_{d, d}$, mean delay time for a new voice call $W_{n, v}$, mean delay time for a handoff voice call $W_{h, v}$, and mean delay time for a data request $W_{d}$. The simulation is done under the following specifications: a $2 \times 2$ wrapped mesh cell structure (the homogeneous cell assumption makes results under $m \times m$ $(m>2)$ almost same as those done under $2 \times 2$ wrapped mesh cell structure; therefore, we adopt $2 \times 2$ rather than others for saving simulation time.) is used with the total available channels $C=7$, voice buffer size $B_{v}=7, K=3$, $\mu_{v}=1, \mu_{d}=100 \mu_{v}$, and $\lambda_{d}=25 \mu_{v}$ in each cell. The detail discussions on the performance of DCA's proposed under different parameters are given as follows.

\section{A. The Effect of Data Queueing}

1) The effect on blocking, forced termination and data dropping probabilities: Figs. 4(a)-4(b) show new voice call blocking and forced termination probabilities as data buffer size ranges from 0 to 25 . For $D C A_{N Q P_{N C}}$, more data requests in the system as data buffer size increases which cause new voice call blocking and forced termination probabilities to increase. However, these two probabilities are less sensitive to data request increase for $D C A_{Q P_{N C}}$ and $D C A_{Q H P_{N C}}$ due to higher priority of voice over data. Fig. 4(c) illustrates that data queueing greatly improves data dropping probability even in cases with priorities.

2) The effect on delays: The phenomena shown in Figs. 4(d)-4(e) are similar to those in Figs. 4(a)-4(b). As for Fig. 4(f), the data delay increases as its buffer size increases.

From the above observations, we know that (i) Although data dropping probability greatly improves due to data queueing, longer delays for data are inevitable. (ii) To gain the benefit of data queueing without degrading QoS of voice service, it needs to set higher priorities to voice service.

\section{B. The Effect of Threshold Control}

1) The effect on the blocking, forced termination and data dropping probabilities: We first note that $T_{v}=B_{v}=7$ indicates that new voice calls and handoff voice calls can be queued once buffer space is available. However, new voice calls can not be queued when the available space is lower than $B_{v}-T_{v}$ while handoff voice calls can still be queued except no available buffer space. Hence decreasing $T_{v}$ makes new voice call blocking increase and improves forced termination probability (shown in Fig. 5(a)). The phenomenon is crystalclear when (new) voice traffic load is high (say, $\lambda=5 \mu_{v}$ ). Since lower value of $T_{v}$ permits fewer queued new voice calls in the system; hence, data packets have a better chance to be served, thus data dropping probability decreases as $T_{v}$ decreases (see Fig. 5(b)).

2) The effect on delays: Smaller value of $T_{v}$ results in more chance to queue handoff request while less chance to queue new voice call request; thus handoff calls incur longer delay than new voice calls and both decrease when $T_{v}$ decreases (see Fig. 5(c)). As for data delay, the results shown in Fig. 5(d) is similar to those in Fig. 5(b).

\section{The Effect of Channel Reservation}

1) The effect on blocking, forced termination and data dropping probabilities: Table I indicates the effect of channel reservation. Obviously, new voice call blocking and data dropping probabilities are dramatically increased by increasing $C_{G}$ 


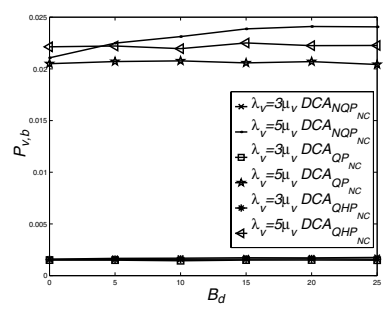

(a)

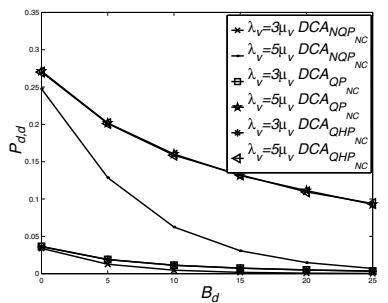

(c)

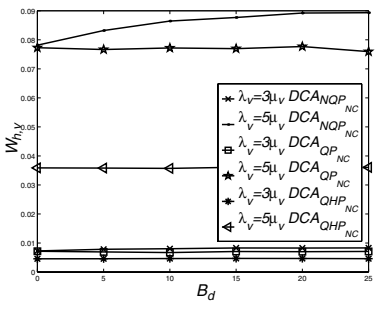

(e)

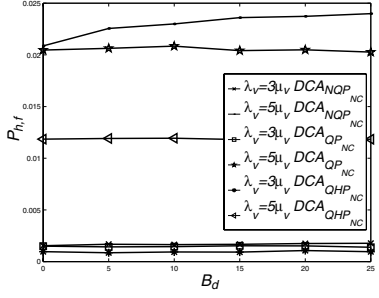

(b)

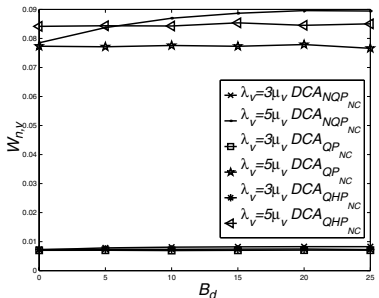

(d)

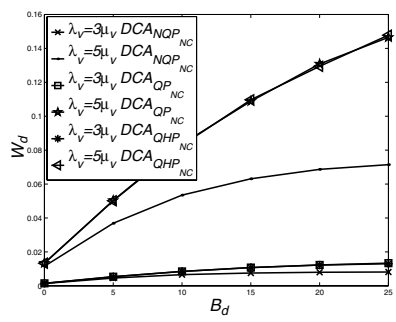

(f)
Fig. 4. The effect of data queueing under $\eta=0.2 \mu_{v}$ with two different (new) voice arrival rates: (a) blocking probability, (a) forced termination probability, (c) data dropping probability, (d) delay for new voice call, (e) delay for handoff voice call, (f) delay for data.

mainly due to poor channel utilization if a portion of channels is dedicated to handoff calls only.

2) The effect on delays: As for delays, the result is similar to the above description pertinent to blocking and forced termination probabilities.

\section{Comparison Among Different Schemes}

1) Observation under various traffic load: When $\lambda_{v} / \mu_{v}<$ 0.3 , all schemes are almost distinguishable; on the other hand, when $\lambda / \mu_{v}>0.3$ differences among different schemes are obvious. From Fig. 6(a), the best to worst schemes on voice blocking probability are $D C A_{Q P_{N C}}, D C A_{Q H P_{N C}}$, $D C A_{N Q P_{N C}}, D C A_{Q P_{T C}}$, and $D C A_{Q P G_{N C}}$. From Fig. 6(b), the best to worst on forced termination are $D C A_{Q H P_{N C}}$, $D C A_{Q P_{T C}}, D C A_{Q P G_{N C}}, D C A_{Q P_{N C}}$, and $D C A_{N Q P_{N C}}$. From Fig. 6(c), $D C A_{N Q P_{N C}}$ makes data dropping the best

TABLE I

THE EFFECT OF CHANNEL RESERVATION UNDER $\lambda_{v}=3.5 \mu_{v}, \eta=0.2 \mu_{v}$, AND $B_{d}=21$.

\begin{tabular}{ccccccc}
\hline$C_{G}$ & $P_{v, b}$ & $P_{h, f}$ & $P_{d, d}$ & $W_{n, v}$ & $W_{h, v}$ & $W_{d}$ \\
\hline 1 & 0.0075 & 0.0018 & 0.0370 & 0.0345 & 0.0079 & 0.0624 \\
2 & 0.0181 & 0.0014 & 0.1099 & 0.0832 & 0.0056 & 0.1432 \\
3 & 0.0420 & 0.0012 & 0.2790 & 0.1880 & 0.0048 & 0.3225 \\
4 & 0.0858 & 0.0012 & 0.5455 & 0.3712 & 0.0043 & 0.7707 \\
\hline
\end{tabular}

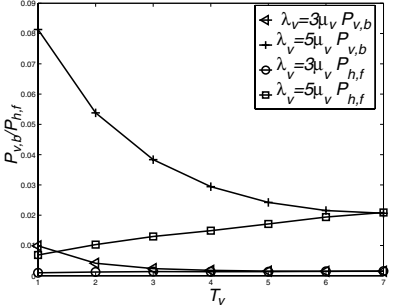

(a)

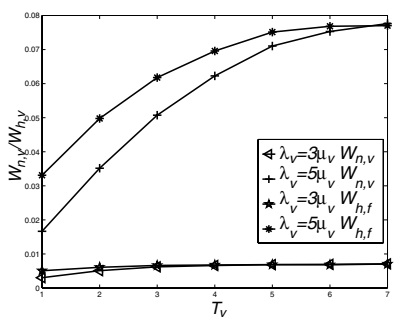

(c)

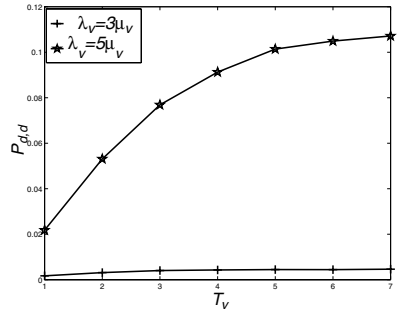

(b)

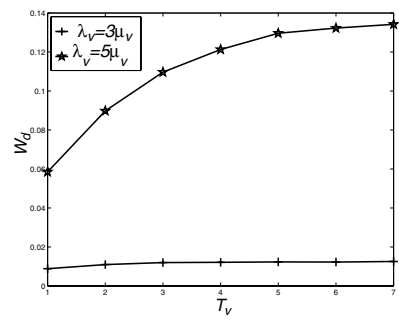

(d)
Fig. 5. The effect of threshold control for the voice buffer $\left(D C A_{Q P_{T C}}\right)$ under $\eta=0.2 \mu_{v}$ and $B_{d}=21$ with two different (new) voice arrival rates: (a) voice blocking and forced termination, (b) data dropping probability, (c) delay for voice calls, (d) delay for data.

performance while $D C A_{N Q P_{N C}}$ performs worst with respect to data dropping. As for delays, the phenomena are analogous to those in Figs. 6(a)-6(c) except (i) $D C A_{Q P_{T C}}$ has best performance on new voice call delay. (ii) For forced termination probability, $D C A_{Q P G_{N V}}$ and $D C A_{Q P_{T C}}$ have reverse results.

2) Observation under various mobility rates: We summarize the following observations: (i) From Figs. 7(a)-7(f), we note that $D C A_{Q P G_{N C}}$ makes smaller forced termination probability/delay but the largest new voice blocking probability and data dropping for new voice call and data service compared to other schemes. (ii) $D C A_{Q H P_{N C}}$ also has better termination probability comparable to $D C A_{Q P G_{N C}}$, however, it degrades less QoS on new call delay/ blocking probability and data delay/ dropping probability. From the above observations, we suggest scheme $D C A_{Q H P_{N C}}$ but not $D C A_{Q P G_{N C}}$ to system designers.

\section{Conclusions}

We have examined the improvements of channel allocation schemes using techniques such as channel reservation, service priority, and queueing with/without threshold control in detail through thorough simulation experiments. The effect on the system performance of these techniques when considering for a channel allocation scheme is drawn as follows: 1) To differentiate the QoS of original and handoff requests, the threshold control is an effective approach to reduce forced termination and data dropping probabilities when queueing is under consideration and voice traffic load is large. But it enlarges new voice call blocking probability. 2) The improvement caused by service priority is impressive on handoff voice call delay and forced termination probability without dramatically degrading QoS of new voice call and data service. 3) Although reservation approach improves forced termination 


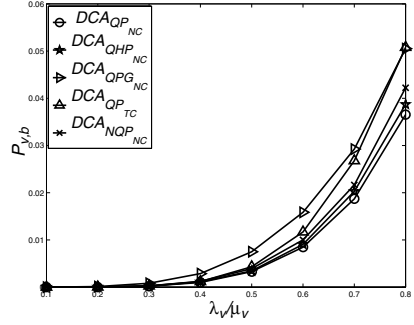

(a)

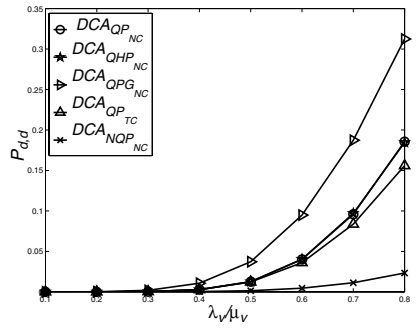

(c)

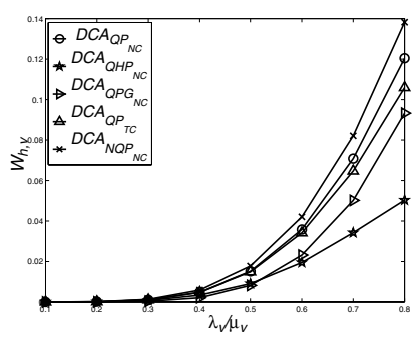

(e)

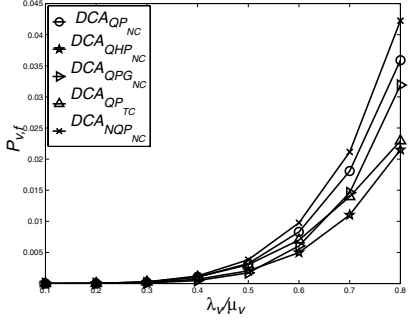

(b)

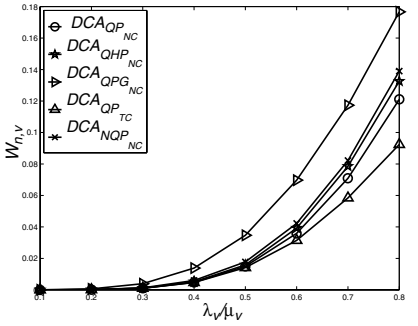

(d)

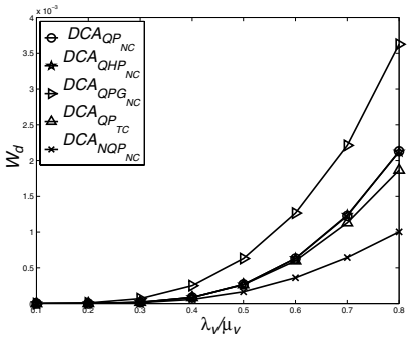

(f)
Fig. 6. Schemes comparison observed for various traffic load under $\eta=$ $0.2 \mu_{v}, T_{v}=4, C_{G}=1$, and $B_{d}=21$ : (a) blocking probability, (a) forced termination probability, (c) data dropping probability, (d) delay for new voice call, (e) delay for handoff voice call, (f) delay for data.

probability and delay time for handoff voice call, it causes the performance of new voice call and data service poor due to inefficient channel utilization. With the observations obtained in this paper, we suggest scheme $D C A_{Q H P_{N C}}$ and $D C A_{Q P_{T C}}$ to be used in the GSM/GPRS system.

Current work can be extended in the following directions in the future: to incorporate the analytical approach to have a quick tool on system performance and to consider the heterogeneous cells etc.

\section{REFERENCES}

[1] R. Beraldi, S. Marano, and E. Palumbo, "Analysis of new priority queuing strategies for handoff and originating calls in mobile cellular radio systems," in Proc. IEEE Wireless Communication System, pp. 6369, 1995.

[2] S. Boumwrdassi, "Dynamically adaptive channel reservation scheme in cellular netwrks," in Proc. IEEE ICPWC 'O0, pp. 269-272, 2000.

[3] C. J. Chang, T. T. Su, and Y. Y. Chiang, "Analysis of a cutoff priority cellular radio system with finite queueing and reneging/dropping," IEEE/ACM Trans. Networking, vol. 2, no. 2, pp. 166-175, Apr. 1994.

[4] J. Eberspacher, H. J. Vogel, and C. Bettstetter, GSM - Switching, Services, and Protocols. New York: Wiley, 2001.

[5] ETSI, "Digital cellular telecommunications system (Phase 2+): General Packet Radio Service (GPRS); Overall description of the GPRS radio interface; Stage 2 (GSM 03.64 version 7.0.0 release 1999)," ETSI/TC, Tech. Rep. Rec. GSM 03.64, 1999.

[6] V. K. Garg, P. E. S. Halpern, and K. F. Smolik, "Third generation (3G) mobile communications systems," in Proc. IEEE ICPWC '99, pp. 39-43, 1999.

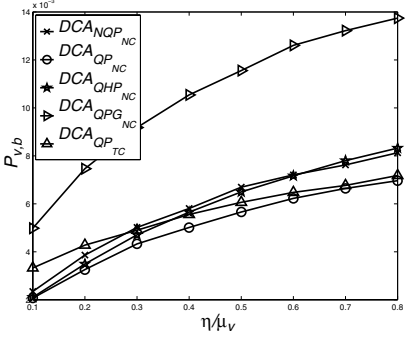

(a)

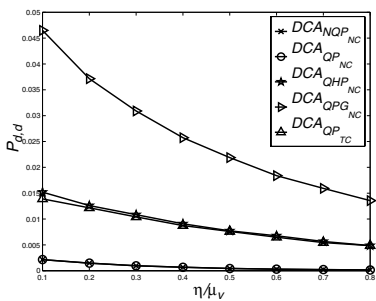

(c)

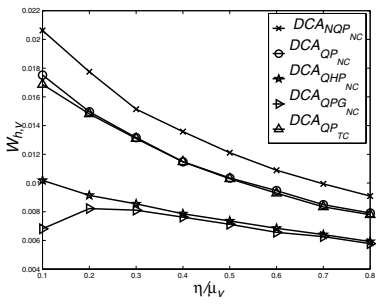

(e)

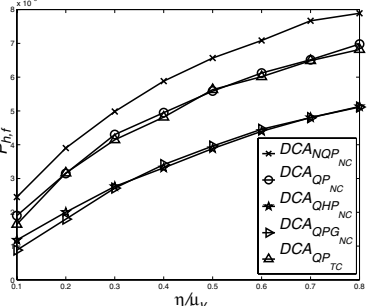

(b)

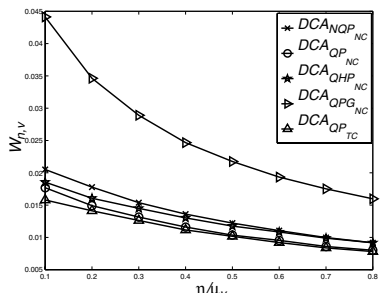

(d)

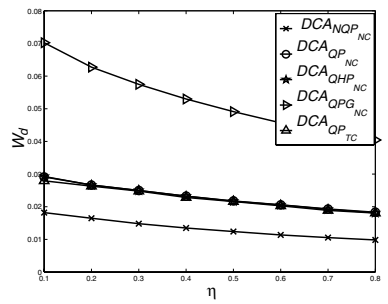

(f)
Fig. 7. Schemes comparison observed for various mobility rates $\eta$ under $\lambda_{v}=3.5 \mu_{v}, T_{v}=4, C_{G}=1$, and $B_{d}=21$ : (a) blocking probability, (a) forced termination probability, (c) data dropping probability, (d) delay for new voice call, (e) delay for handoff voice call, (f) delay for data.

[7] R. A. Guerin, "Queueing-blocking system with two arrival streams and guard channels," IEEE Trans. Commun., vol.36, no. 2, pp. 153-163, 1988.

[8] C. Lindemann and A. Thummler, "Performance analysis of the general packet radio service," in Proc. IEEE Distributed Computing Systems, pp. 673-680, 2001.

[9] P. Lin and Y. B. Lin, "Channel allocation for GPRS," IEEE Trans. Vehicular Tech., vol. 50, pp. 375-387, 2001.

[10] D. E. McDysan and D. L. Spohn, ATM Theory and Application. New York: McGraw-Hill, 1995.

[11] A. G. Qureshi and A. S. Ween, "Dynamic resource allocation for GSMGPRS services over a LEO satellite system," in Proc. IEEE ICICS '97, pp.20-24, 1997.

[12] Y. Raivio, "4G - hype and reality," in Proc. IEE 3 G Mobile Communication Technologies '01, pp. 346-350, 2001.

[13] M. Rajaratnam and F. Takawira, "A single cell model for the performance analysis of the radio layer in GSM Phase 2+ (GPRS) networks under voice and data traffic," in Proc. IEEE PIMRC '01, pp.118-123, Sept. 2001

[14] R. Steele, "Beyond 3G," in Proc. IEEE Broadband Communications '00, pp. 1-7, 2000.

[15] P. Stuckmann and F. Muller, "GPRS radio network capacity and quality of service using fixed and on-demand channel allocation techniques," in Proc. IEEE VTC 'O0, pp. 440-444, 2000.

[16] S. Tekinary and B. Jabbari, "Handover and channel assignment in mobile cellular networks," IEEE Commun. Mag., pp. 42-46, Nov. 1991.

[17] A. Tripathi and K. N. Sivarajan, "Resource allocation in GPRS wireless networks," in Proc. IEEE ICPWC '00, pp. 388-394, 2000.

[18] J. Wang, Q. A. Zeng, and D. P. Agrawal, "Performance analysis of preemptive handoff scheme for integrated wireless mobile networks," in Proc. IEEE GLOBECOM '01, pp. 3277-3281, 2001.

[19] A. Xhafa and O. K. Tonguz, "Dynamic priority queuing of handoff requests in PCS,' in Proc. IEEE ICC '01, pp. 341-345, 2001. 